\title{
Knee Salvation Procedure (Arthroscopic Debridement Combined with LP-PRP Intraarticular Injection and Proximal Fibular Osteotomy) vs Proximal Fibular Osteotomy Alone in the Management of Medial Compartment Knee Osteoarthritis: A Comparative Study of 60 Cases
}

\author{
Gourav Mazumdar', Vineet Kumar Arora', S. K Sharma ${ }^{3}$, Garv Arora ${ }^{4}$, \\ Vikas Chouhan ${ }^{5}$
}

\author{
${ }^{1}$ DNB Resident, Orthopedics, DDU Hospital New Delhi. \\ ${ }^{2}$ Senior Specialist, Orthopedics, DDU Hospital, New Delhi. \\ ${ }^{3}$ Head of Department, Orthopedics, DDU Hospital, New Delhi. \\ ${ }^{4}$ Data compilation \& Analysis, High School Student, BGSIPS, Dwarka, New Delhi. \\ ${ }^{5}$ DNB Resident, Orthopedics, DDU Hospital New Delhi.
}

Corresponding Author: Vineet Kumar Arora

DOI: https://doi.org/10.52403/ijrr.20220245

\begin{abstract}
Objective: The study was performed to compare the outcomes of Knee salvation procedure (KSP) and Proximal Fibular Osteotomy (PFO) in medial compartment osteoarthritis (OA) of knee joint. Knee salvation procedure includes Arthroscopic lavage and debridement combined with Leucocyte poor platelet rich plasma [LPPRP] application and Proximal fibular osteotomy.

Material and Methods: 60 patients with medial compartment osteoarthritis of knee joint were selected prospectively and randomly allocated into knee salvation procedure (KSP) group (30 patients, observation group) and PFO group (30 patients, control group). The follow up period was 1 year and outcomes were compared between the two groups.

Results: VAS score improved in patients postoperatively and was settled to lower values by the end of 1 year of follow up ( $\mathrm{P}<0.005)$. The mean Knee score rating of patients Pre operatively was $53.1 \pm 6$ and $75.4 \pm 10.16$ at the end of 1 year postoperative follow up. The mean function score pre operatively was $46.5 \pm 13.84$ which improved to 71.50 at the end of 1 year. The majority of patients who were with normal
\end{abstract}

Body mass index had good to excellent results. The mean ratio MJS/LJS Post operatively improved for KSP and PFO group ( $\mathrm{P}$ - value $<0.0001)$

Conclusion: In medium to long term KSP has superior effects than PFO in improving the VAS score and KSS while PFO has better effects in immediate postoperative to short term. BMI also has implication in outcome of procedures with better results in normal BMI patients in KSP group.

Key Words: osteoarthritis (OA), Proximal Fibular Osteotomy (PFO), LP-PRP, Knee Salvation Procedure, Visual Analogue Scale (VAS), Medial Joint Space (MJS), Lateral Joint Space (LJS), Knee Society Score (KSS), Body mass index (BMI)

\section{INTRODUCTION}

Osteoarthritis (OA) knee is a wellknown degenerative joint disease. It is the mechanical abnormalities that leads to wear and tear of joints, articular cartilage and subchondral bone ${ }^{1}$. It is a very common joint disorder affecting the elderly population throughout the world. Now a 
Gourav Mazumdar et.al. Knee salvation procedure (arthroscopic debridement combined with LP-PRP intraarticular injection and proximal fibular osteotomy) vs proximal fibular osteotomy alone in the management of medial compartment knee osteoarthritis: A comparative study of 60 cases.

days overweight and obese people commonly suffer from OA. According to worldwide estimate $9.6 \%$ of men and $18 \%$ of women over 60 years have symptomatic $\mathrm{OA}^{2}$. Nowadays there is an increasing trend of involvement of relatively younger population being affected from this disease may be due to change in the lifestyle. These subsets of individuals are in the watershed area of getting treatment and generally either treated only by analgesics or over enthusiastically treated by knee arthroplasty. The radiographic classification of osteoarthritis knee is based either on the degree of joint space narrowing, as with the Ahlback grading, or on the presence of osteophytes, such as the Kellgren and Lawrence classification ${ }^{3}$ and international knee Documentation committee (IKDC) grading.

There are numerous treatment modalities for osteoarthritis knee joint ranging from conservative management in the form of physical therapies, analgesics, viscosupplementations intraarticularly to surgical interventions like osteotomies around the knee joint, arthroscopic procedures and knee arthroplasties. Not a single method can be considered a gold standard for a particular patient because of the different clinical picture of the same disease process in different individuals and further there is considerable growing trend to treat most of the moderate to severe cases by knee arthroplasty.

Non-surgical management include lifestyle modification, physiotherapy, orthosis, pharmacotherapy is still the primary mode of treatment while surgical management including joint preserving arthroscopic procedures (e.g. debridement, lavage, synovectomy, etc), joint alignment procedures (e.g. High Tibial osteotomy, Distal femoral osteotomy and Proximal fibular osteotomy) and joint replacement (Unicondylar knee arthroplasty and Total knee arthroplasty) ${ }^{4}$. Total knee arthroplasty (TKA) is the main stay of treatment for advance disease. It aims to relieve pain, improve joint function and mobility is the main surgical alternative in severe osteoarthritis knee patients. However, TKA is an expensive and complex procedure having its own complications, and some patients need a revision surgery ${ }^{5}$. In developing countries like India where it is difficult to imagine knee replacement to every OA knee patient especially those in early stages and failed with symptomatic management, a new treatment alternative (Knee Salvation Procedure) is required which if offered to those patients with early stages of OA knee, which should be cost effective and readily available to everyone and can delay the future need of TKA. Compared with TKA, Proximal Fibular Osteotomy (PFO) is simple, safe, affordable surgery and does not require any implant insertion and relieves pain by redistributing the body weight on the lateral and medial tibial plateau after surgery. The principle of fibular osteotomy originates from the theory of "non-uniform settlement", which suggest a significant settlement of the tibial plateau following osteotomy. Proximal fibular osteotomy weakens the lateral fibular support and leads to a correction of the Varus deformity, which can subsequently shift the loading force from the medial compartment more laterally, leading to decreased pain and a satisfactory functional recovery.

PRP consists of a volume of plasma with a platelet concentration above whole blood baseline values ${ }^{7}$. Based on the bioactive molecules that platelet release after activation and the regenerative and healing potential they have, the use of PRP has been increasing for various degenerative diseases. Many preclinical and randomized control studies (RCT) provided strong evidence for clinical improvement and pain relief by intraarticular PRP application, even in unicondylar knee arthroplasty $^{8}$.The supra-physiological release of plateletderived factors directly at the site of morbid cartilage, particularly with interest to knee OA, may stimulate the natural regenerative signalling cascade and enhance the healing of tissue with further mediation of the anti- 
Gourav Mazumdar et.al. Knee salvation procedure (arthroscopic debridement combined with LP-PRP intraarticular injection and proximal fibular osteotomy) vs proximal fibular osteotomy alone in the management of medial compartment knee osteoarthritis: A comparative study of 60 cases.

inflammatory response ${ }^{9}$.We thought that additional peri operative LP-PRP intraarticular injection may be a promising step because of its frequent use in management of $\mathrm{OA}$ and encouraging results in literatures.

Arthroscopic joint debridement includes smoothening of cartilage lesions, meniscectomy, loose body removal, ligament release and synovectomy. This treatment deals with the removal of mechanical hindrances, the improvement of gliding within the cartilage layers and/or the release of shrunken ligaments. Because every arthritic joint has its own spectrum of damage, an individual treatment approach is required. It is more than just a simple "chondro-saving" procedure ${ }^{10}$ and can be combined with procedures like osteotomies (PFO or HTO) or cartilage restoring techniques (drilling, abrasion, micro fracturing and autologous chondrocyte implementation).

This study aims to analyse whether outcomes of proximal fibular osteotomy (PFO) combined with arthroscopic joint lavage with debridement and application of leucocyte poor PRP (Knee Salvation Procedure, KSP) are better than proximal fibular osteotomy alone in the management of medial compartment knee osteoarthritis patients as sufficient literature is not available where the three procedures (arthroscopic joint lavage and debridement, PRP application and PFO) have been done simultaneously in the same sitting.

Osteoarthritis knee has been extensively classified in the past. Currently, the most common radiological classification used is Ahlback Classification Proposed by Ahlback et al $^{11}$. in 1968 based on the joint space and bone attrition. Another radiological classification for OA knee is Kellgren Lawrence classification ${ }^{12}$. International Knee Documentation committee (IKDC) classification For the grading of osteoarthritis in the knee ${ }^{13}$.

\section{MATERIALS AND METHODS}

Preoperative assessment was done by taking detailed history regarding the site of pain i.e. single or both knees involved, duration of pain, aggravating and relieving factors, distance of symptom free walking, stair climbing, with or without use of railing of the stairs, activity of daily living, and patients with history of multiple joint involvement, significant trauma, any joint swelling, loss of weight and appetite, these patients were excluded as these patients were having secondary knee osteoarthritis. Physical examination was done and checked for limb deformity, joint effusion, joint line tenderness, range of motion and ligament instability. Patients were evaluated with Visual Analog score (VAS), Knee society score (KSS), Radiographs of both knee joints in weight bearing Anterior-posterior and Lateral view. The medial joint space and the lateral joint space and ratio of medial and lateral joint space were calculated on standing weight-bearing radiograph. Then knee joint $\mathrm{OA}$ was classified as per IKDC classification. Preoperative investigations like hemogram, random blood sugar, liver and kidney function test, viral markers and electrocardiogram were done. The preanaesthetic check-up was done and fit patients were subsequently admitted for surgery.

OPERATIVE PROCEDURE: After spinal anaesthesia was given surface marking of anatomical structures including borders of the patella, tibial tubercle, and patellar tendon, the medial and lateral tibial joint line, and the head of the fibula were marked on the skin for placement of portals. The diagnostic arthroscopy was done after conventional arthroscopic portals were made. Joint debridement was done with help of shaver and fibrillations and frayed menisci and cartilage debrided and a thorough joint lavage done. After completing arthroscopic interventions by lavage, cartilage debridement irrigation fluid was evacuated. 
Gourav Mazumdar et.al. Knee salvation procedure (arthroscopic debridement combined with LP-PRP intraarticular injection and proximal fibular osteotomy) vs proximal fibular osteotomy alone in the management of medial compartment knee osteoarthritis: A comparative study of 60 cases.

Before the surgery was started, 15 $20 \mathrm{ml}$ of blood was already collected from a peripheral vein in EDTA tube and was centrifuged for 5 minutes with $1800 \mathrm{rpm}$ and separated into three fractions, with the thrombocyte phase being isolated in a second sterile syringe and leucocyte poor platelet rich plasma (LP-PRP) was prepared. Initially we injected the leucocyte poor platelet rich plasma to the knee joint directly from the anterolateral portal but this sometimes leads to oozing out of the LPPRP from the portal. So, another alternative was used to inject the LP-PRP intraarticularly after the arthroscopy instruments were removed and portals were stitched. After arthroscopic lavage and debridement and LP-PRP application proximal fibular osteotomy was done. A 5$8 \mathrm{~cm}$ lateral skin incision was taken $6-8 \mathrm{~cm}$ below fibular head over the posterior border of fibula. Haemostasis was achieved by using electro cautery. The fascia was incised in the line with the septum between the peroneus and soleus muscle and the deep fascia was split in this line. The muscles were subperiosteally elevated from the fibula to expose the shaft of fibula. The distal and proximal osteotomy sites were identified that was 1 to $2 \mathrm{~cm}$ of the fibular shaft and drill holes were made using 2.5 $\mathrm{mm}$ drill bit or $2.5 \mathrm{~mm}$ Kirschner wire. The osteotomy was made at the distal and proximal sites using an oscillating saw or osteotome and hammer. The osteotomized segment was removed. Thee bone ends were sealed with bone wax to prevent postoperative bleeding. A through wound wash was given and the incised wound was closed in layers. Compression bandage was used. Standard post-operative protocol of the institute was followed. In the control group we did only proximal fibular osteotomy following the same preoperative and anaesthetic protocols.

Postoperative Management: Intravenous antibiotics including injection ceftriaxone 1 gm and injection amikacin 500mg were given for 3 days and then changed into oral antibiotics for 5 to 7 days. The limb was elevated and active toe and ankle movements were encouraged. Patients were encouraged for early active hip, knee and ankle range of motion exercise, as soon as patients were comfortable. Patients were kept non weight bearing for 24-48 hrs and gradual and partial weight bearing was allowed after that as per pain tolerance. Post-operative dressing was done on 2 nd, 5th, 10th and 14th days of surgery. Suture was removed after 12 to 14 days of surgery. Post-operative radiographs were done.

Follow up and evaluation: Patients were evaluated at routine intervals of 2 weeks, 1 month, 3 months and 6 months and 1 year. At every visit they were assessed for Visual analogue score, Knee Society Score, and a Standing Weight bearing anterior-posterior radiograph of the bilateral knee joint. On the radiograph, Medial and Lateral joint spaces, ratio of medial to lateral joint space were calculated. A watchful eye was kept for any complications especially in the immediate postsurgical period and also in the follow-up visits

\section{RESULTS AND OBSERVATIONS}

Most of the patients who participated in the study were in the normal BMI group out of which $70 \%$ were in KSP and $63.33 \%$ in PFO group. $13.33 \%$ patients in KSP and $26.33 \%$ patients in PFO group were underweight while overweight patients in KSP and PFO group were $16.66 \%$ and $10 \%$ respectively.

The mean pre operative VAS in the patients of KSP was 8.73 \pm 0.45 , at immediate post op $4.63 \pm 0.85$, at 1 month post operative was $4.1 \pm 0.23$ and past 1 year was $2.67 \pm 1.24$. p- value was $<0.0001$, which was statistically significant. 
Gourav Mazumdar et.al. Knee salvation procedure (arthroscopic debridement combined with LP-PRP intraarticular injection and proximal fibular osteotomy) vs proximal fibular osteotomy alone in the management of medial compartment knee osteoarthritis: A comparative study of 60 cases.

\section{COMPARISION OF BMI IN KSP AND PFO}

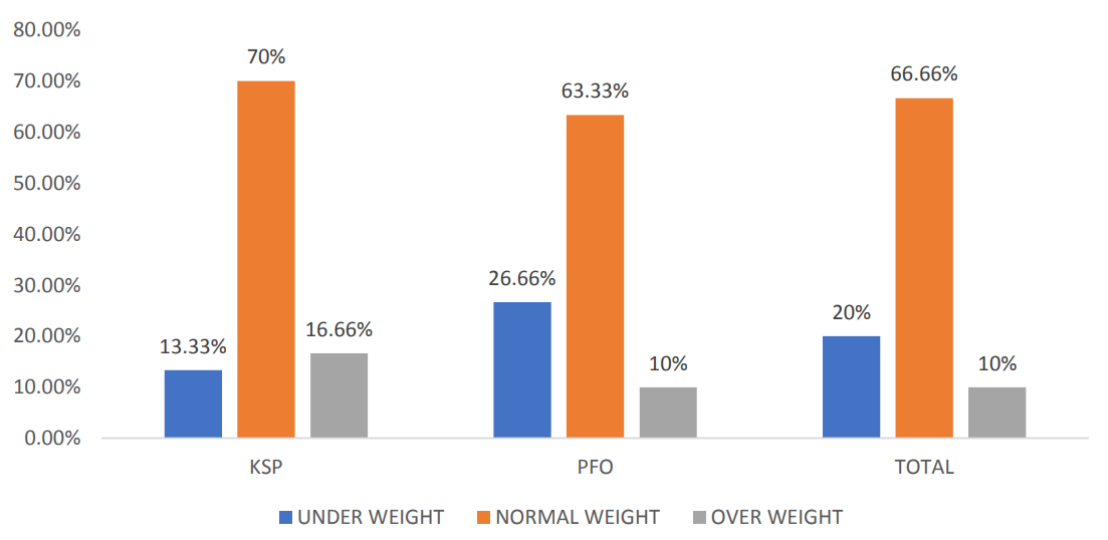

FIGURE 1: Comparison of BMI of patients in KSP and PFO

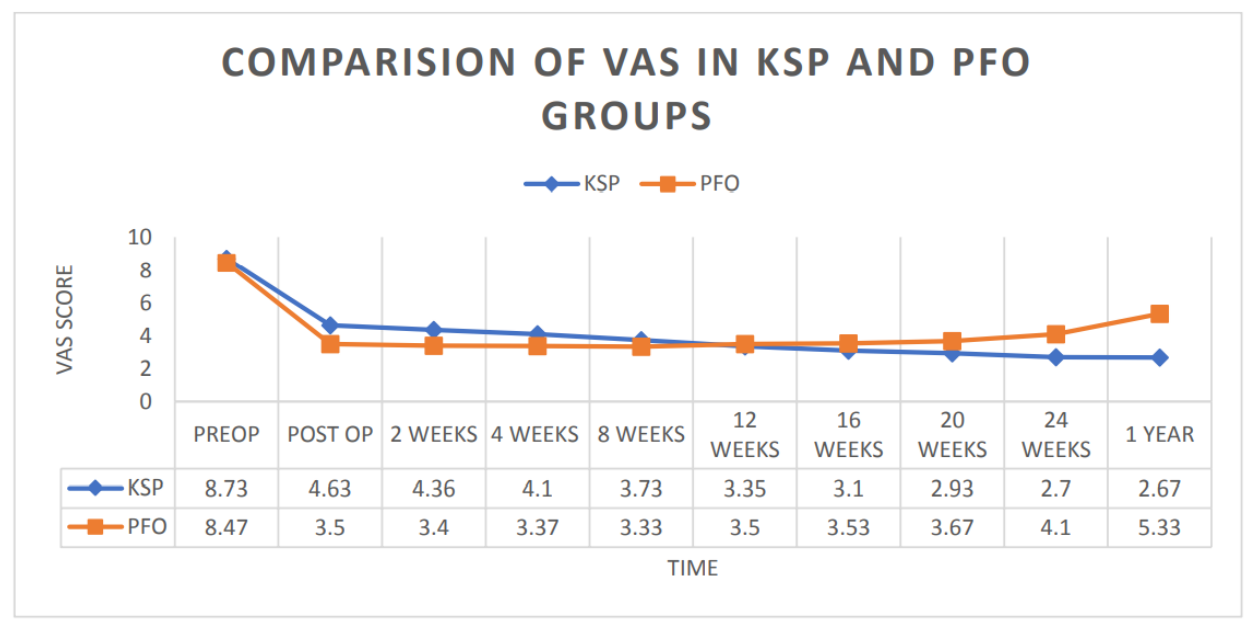

FIGURE 2: Comparison of VAS trend in KSP and PFO group

The mean pain domain score of Knee society score in the KSP group was 12 \pm 6.1 which progressively increased to $35 \pm$ 9.65 at the end of 1 year. In the PFO group the mean pain score preoperatively was
$11.33 \pm 5.71$ which peaked to $36 \pm 8.35$ at postoperative 1 month and then declined to $28 \pm 8.05$ at the end of 1 year. P- value was less than 0.0001 in both the groups which was statistically significant.

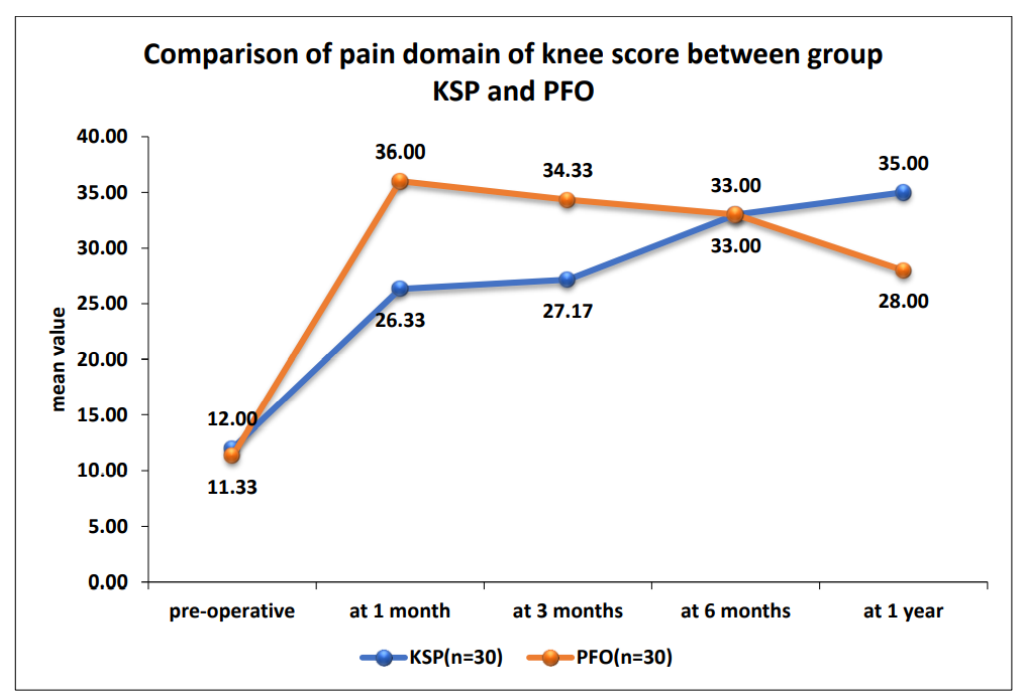

FIGURE 3: Comparison of pain domain of knee score between KSP and PFO group 
Gourav Mazumdar et.al. Knee salvation procedure (arthroscopic debridement combined with LP-PRP intraarticular injection and proximal fibular osteotomy) vs proximal fibular osteotomy alone in the management of medial compartment knee osteoarthritis: A comparative study of 60 cases.

The mean Knee score rating for KSP It peaked to $75.9 \pm 8.59$ at 1 month group patients Pre operatively was $53.1 \pm 6$. It peaked to $75.4 \pm 10.16$ at the end of 1 year. The mean knee score rating for PFO postoperatively and then declined to $67.67 \pm$ 7.91 at the end of 1 month. P- value was group for pre operatively was $51.77 \pm 6.08$. $<0.0001$, which was statistically significant.

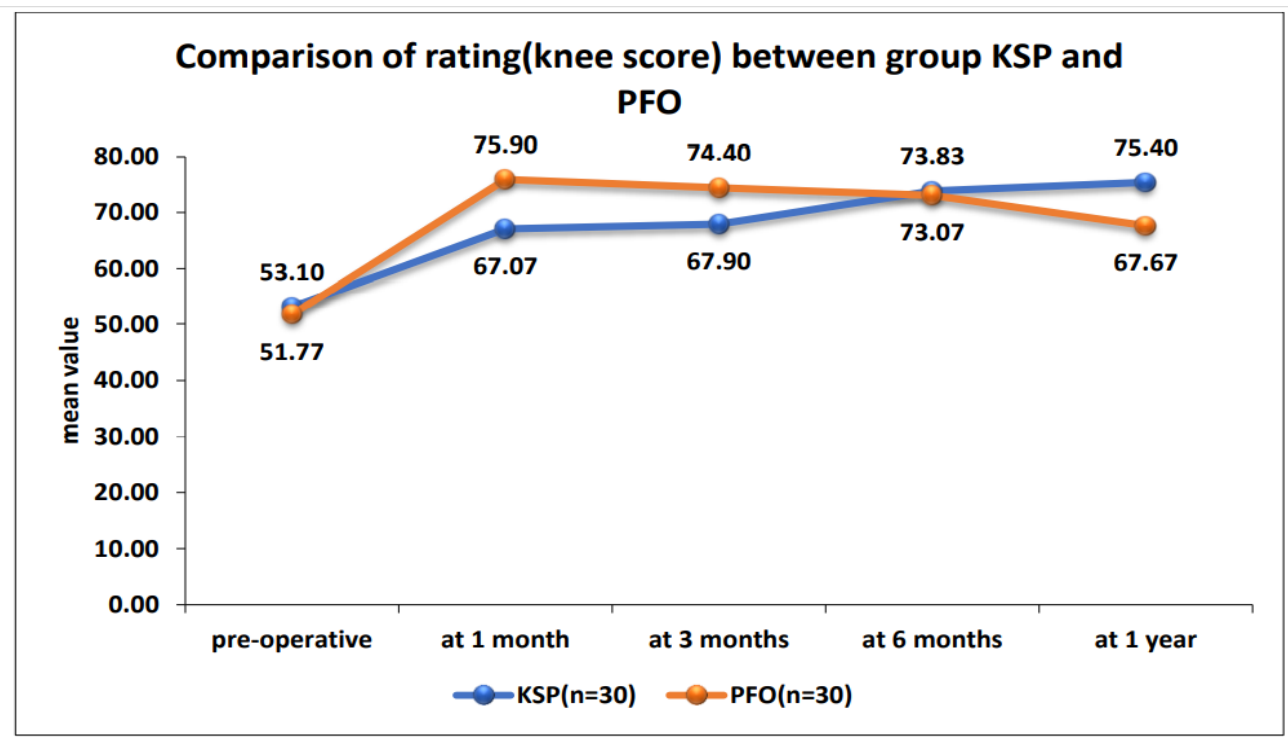

Figure 4: Comparison of knee score between KSP and PFO group

The mean function score preoperatively in KSP group was $46.5 \pm$ 13.84. It improved progressively to 71.50 at the end of 1 year. The mean function score preoperatively in PFO group was $47.17 \pm$ 14.18 which attained its peak at postoperative 1 month but gradually decreased to $62.83 \pm 5.52$ at the end of 1 year. P-value was less than 0.0001 in both the groups which was statistically significant.

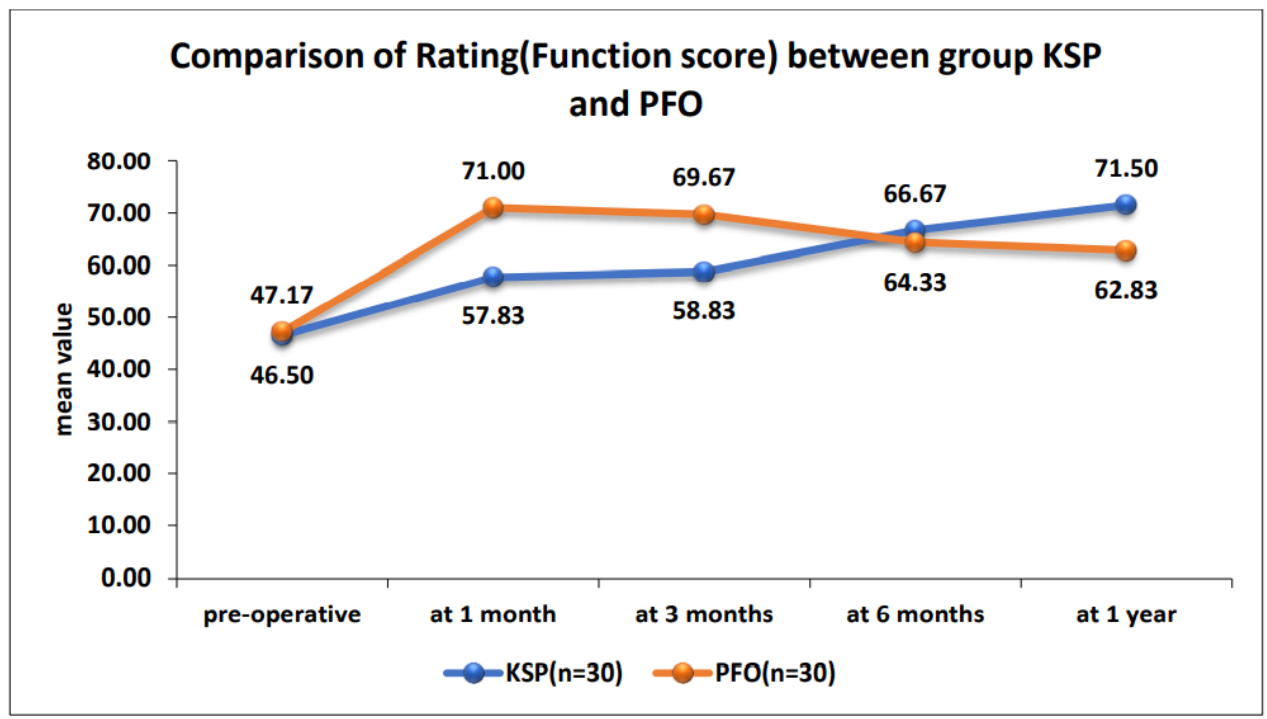

FIGURE 5: Comparison of function score between KSP and PFO group

Number of patients with good scores peaked in both groups at 3 months. Percentage of patients with excellent scores peaked at 1 month in PFO group (47\%) and decreased to $21.67 \%$ patients at the end of 1 year while in the KSP group patients with 
Gourav Mazumdar et.al. Knee salvation procedure (arthroscopic debridement combined with LP-PRP intraarticular injection and proximal fibular osteotomy) vs proximal fibular osteotomy alone in the management of medial compartment knee osteoarthritis: A comparative study of 60 cases.

excellent scores started to increase from 3 term while KSP effects are slower to grow month and peaked to $36.67 \%$ at the end of 1 year. This demonstrates that beneficial effects of PFO are not well sustained in long with respect to PFO but are well sustained at 1 year.
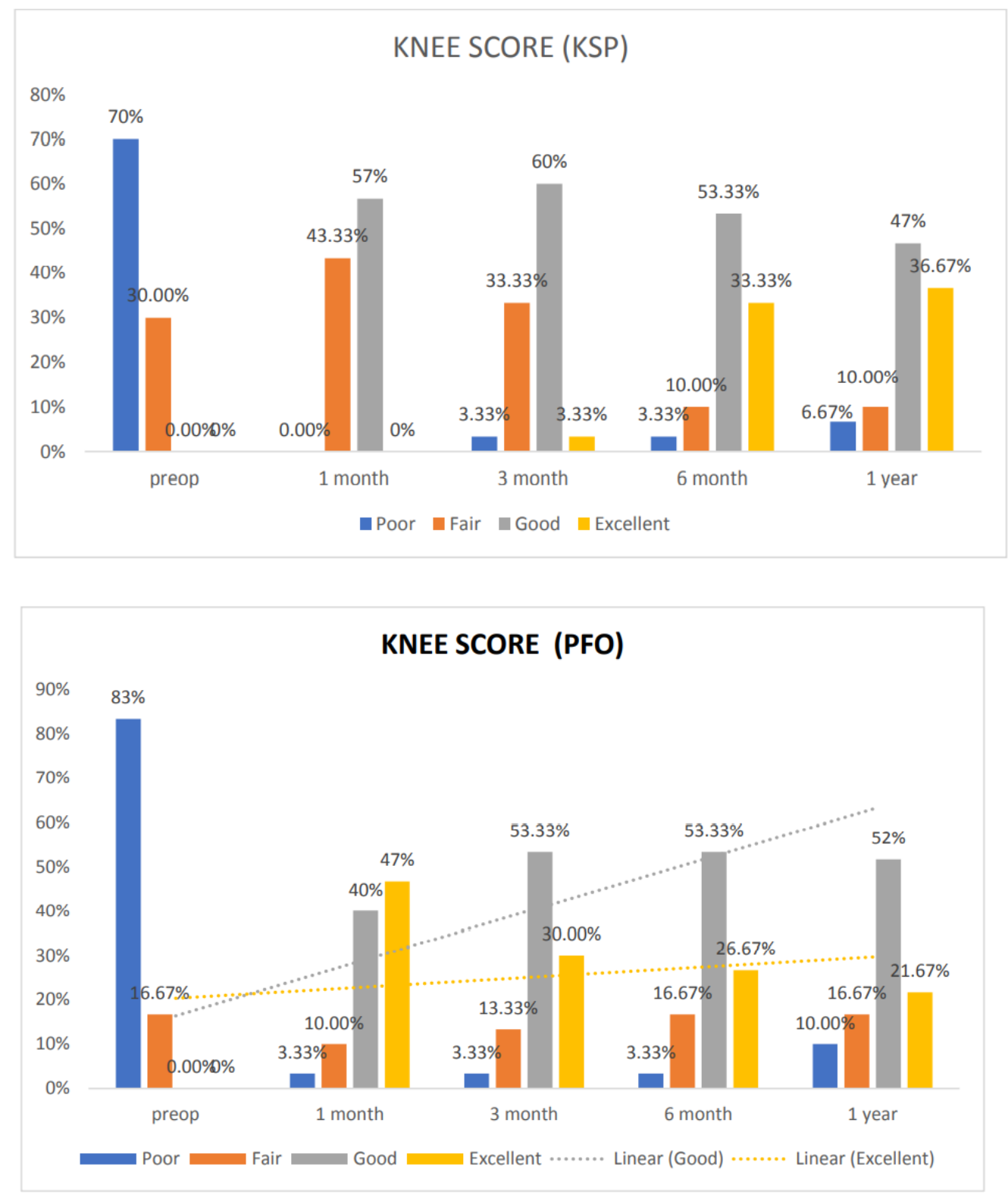

FIGURE 6: Knee score trends in KSP and PFO group

The patients with good function score had an exponential rise in the KSP group from $6.67 \%$ preoperatively to $60 \%$ at the end of 1 year while there was linear rise of the score in the PFO group from $13.33 \%$ preoperatively to $70 \%$ at 1 year. Patients with excellent scores peaked in the PFO group at 1 month but this followed a decreasing trend thereafter. There were no patients with excellent scores in the PFO group at the end of 1 year while in the KSP group $23.33 \%$ patients were having excellent scores. This graph shows KSP has a more beneficial effects on long term which can be attributed to LP-PRP and joint lavage in addition to PFO. 
Gourav Mazumdar et.al. Knee salvation procedure (arthroscopic debridement combined with LP-PRP intraarticular injection and proximal fibular osteotomy) vs proximal fibular osteotomy alone in the management of medial compartment knee osteoarthritis: A comparative study of 60 cases.
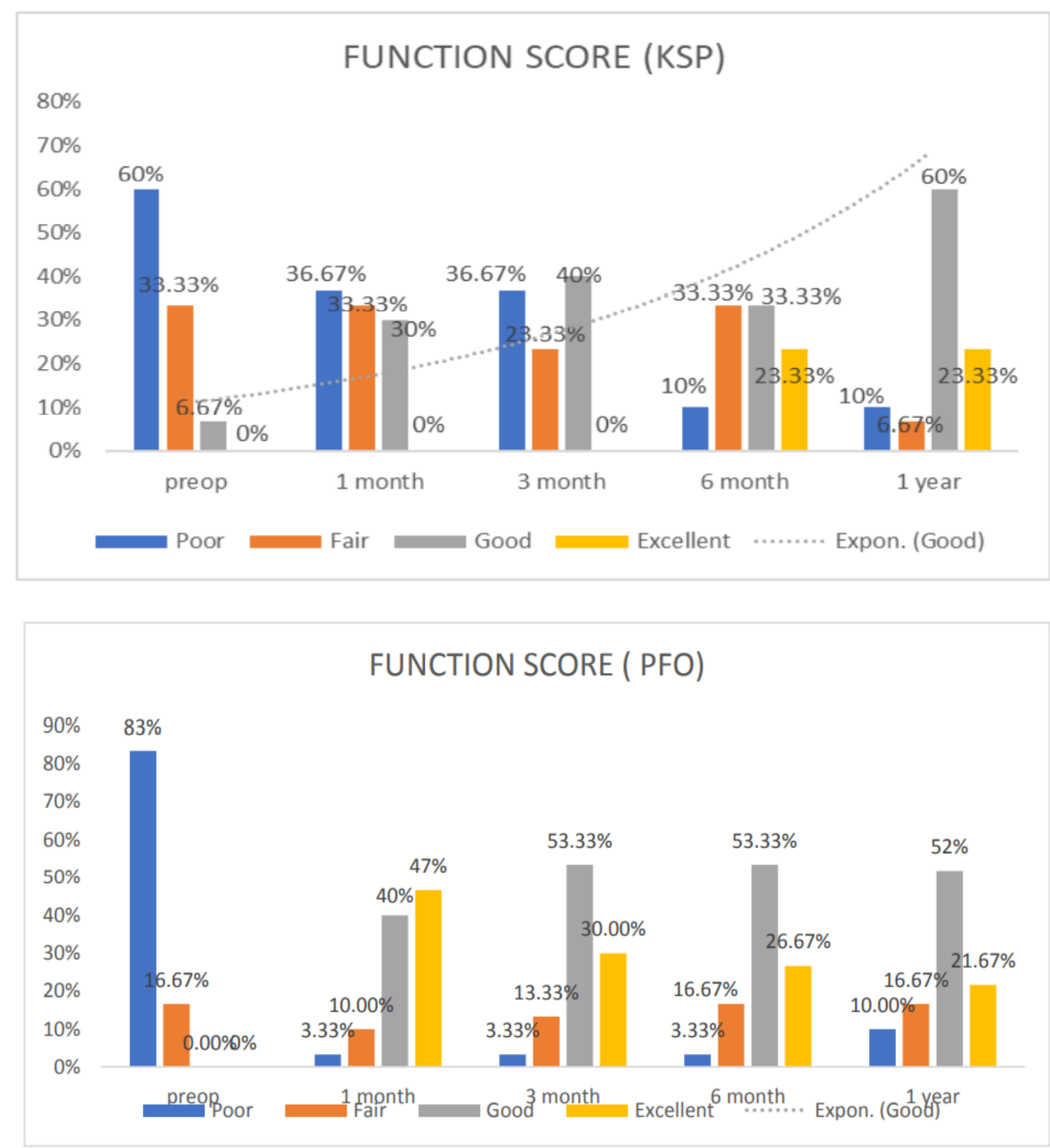

FIGURE 7:Trend of Function score in KSP and PFO group

In the KSP group, the patients with good knee score $(46.66 \%)$ belong to normal BMI while majority of patients with excellent scores $(23.33 \%)$ were having normal BMI. All the patients who were underweight had excellent results $(13.33 \%)$. In the PFO group patients with normal BMI had Good (36.66\%) to fair results $(26.66 \%)$ while underweight patients had good $(20 \%)$ to excellent $(6.66 \%)$ results.
The majority of patients $(50 \%)$ with good function score in KSP group belong to normal BMI while majority of patients $(43.33 \%)$ with normal BMI in PFO had fair results at the end of 1 year. $16.66 \%$ of normal BMI patients and $6.66 \%$ of patients with low BMI had excellent results in KSP group. 
Gourav Mazumdar et.al. Knee salvation procedure (arthroscopic debridement combined with LP-PRP intraarticular injection and proximal fibular osteotomy) vs proximal fibular osteotomy alone in the management of medial compartment knee osteoarthritis: A comparative study of 60 cases.

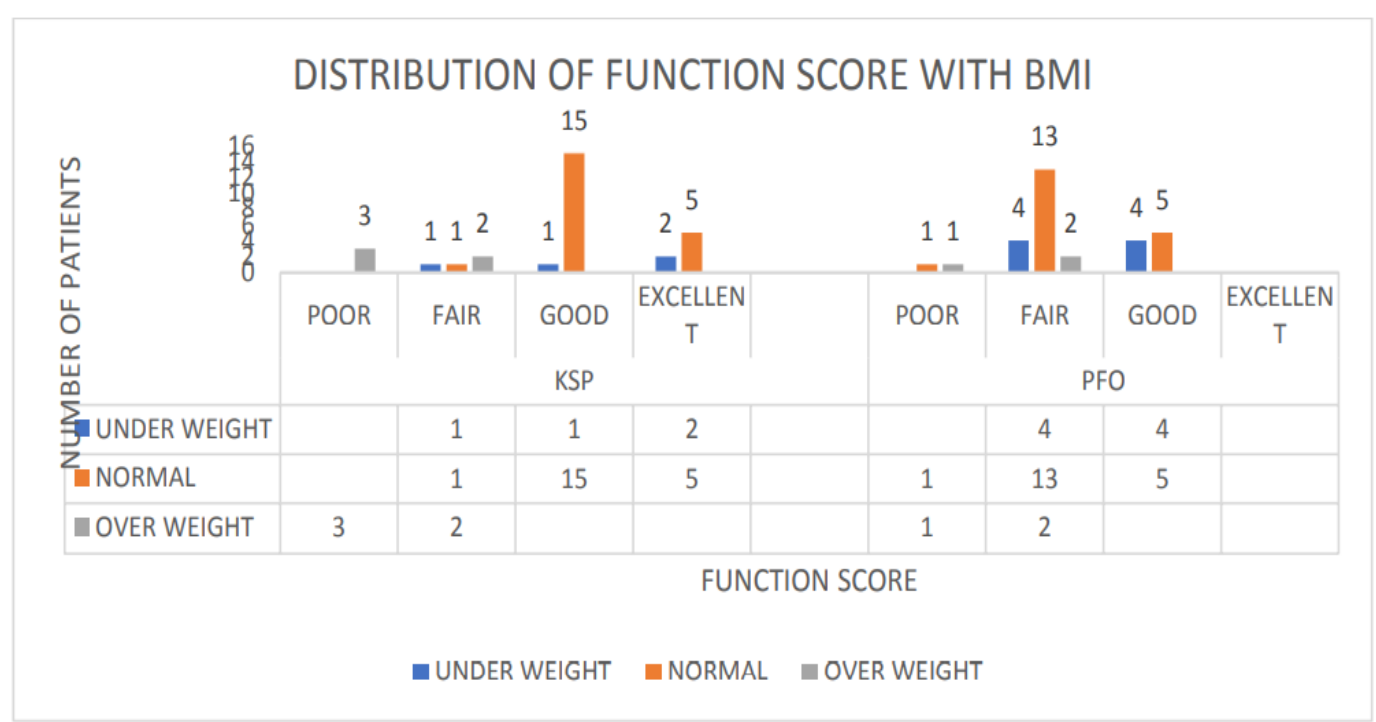

FIGURE 8: Distribution of Function score with BMI

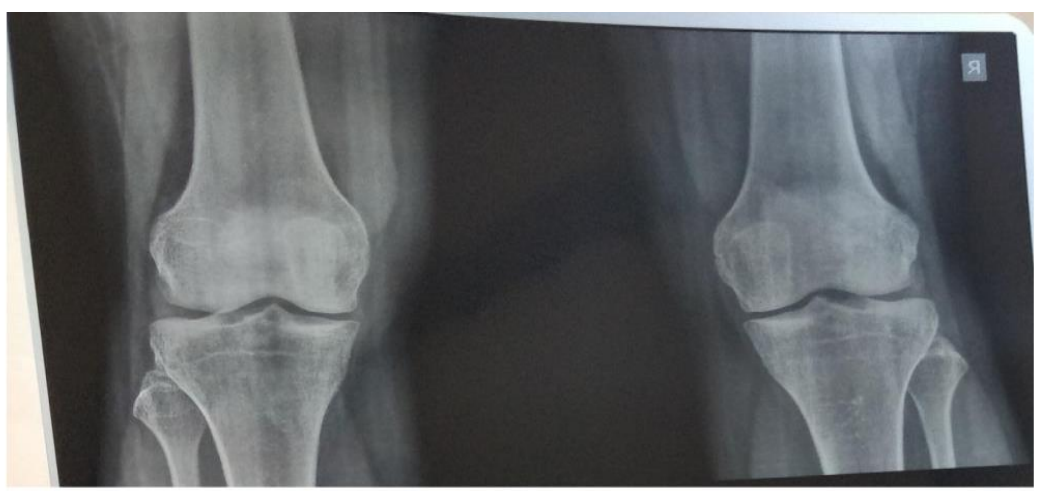

Fig: Pre-operative $x-$ ray of Bilateral knee joint in standing AP View

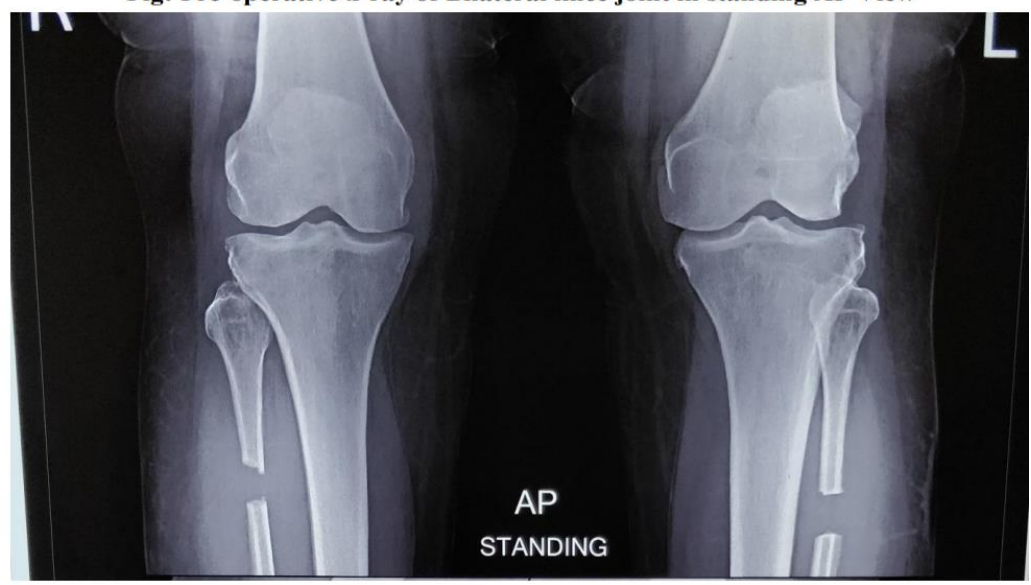

Fig: Bilateral knee radiograph after 12 months post-operative in standing AP View Figure 9: pre operative and post operative $X$ rays

\section{SUMMARY}

- Most of the patients $(46.66 \%)$ of the study belong to the 51-60 years of age group. • Females constituted $68.33 \%$ in the study population. - 43 patients $(71.67 \%)$ in the study had grade 3 osteoarthritis of knee (IKDC). • Follow up period of the study was 12 months. $\bullet$ Mean BMI of the patients $22.77 \pm 77$. Both the procedures gave good to excellent results in patients with low to normal BMI. KSP group patients had slightly better results as compared to PFO group patients with normal BMI. - Mean preoperative VAS in knee salvation group was $8.73 \pm 0.45$ and in PFO group was 8.47 
Gourav Mazumdar et.al. Knee salvation procedure (arthroscopic debridement combined with LP-PRP intraarticular injection and proximal fibular osteotomy) vs proximal fibular osteotomy alone in the management of medial compartment knee osteoarthritis: A comparative study of 60 cases.

\pm 0.51 . The VAS in PFO group had an immediate improvement as compared to KSP group till 12 week and demonstrated rising trend of VAS score thereafter. While patients who underwent KSP had trend of improving VAS score till the end of 1 year. Mean postoperative (1 year) VAS in KSP group was $2.67 \pm 1.24$ and in PFO group was $5.33 \pm 0.76$. $\bullet$ Mean preoperative Pain domain of knee score in KSP group and PFO group were $12 \pm 6.1$ and $11.33 \pm 5.71$ respectively while postoperative (1 year) the score in KSP group and PFO group were 35 \pm 9.65 and $28 \pm 8.05$ respectively. The pain domain score also follows the VAS score trend with an immediate postoperative improvement in PFO group but could not sustain at the end of 1 year while patients treated with knee salvation procedure (KSP) showed consistent improvement in pain domain of knee score. - Mean preoperative Knee score in KSP group and PFO group were $53.1 \pm 6.52$ and $51.77 \pm 6.08$ respectively. The mean post-operative 1 year Knee score were $75.4 \pm 10.16$ and $67.67 \pm 7.91$ respectively with patients with good score in $50 \%$ and excellent score in $36.67 \%$ of patients in KSP and 52\% patients with good and $21.67 \%$ excellent scores in PFO group respectively. 66 - Mean preoperative function score in KSP group and PFO group were $46.5 \pm 13.84$ and 47.17 \pm 14.18 respectively while the mean postoperative function score in KSP group and PFO group $71.5 \pm 12.88$ and $62.83 \pm$ 5.52 respectively. In our study in KSP group we had in $23.3 \%$ excellent result and $60 \%$ patient with good result. In PFO group we had $52 \%$ with good result followed by $21.657 \%$ excellent results. $\bullet$ Only $5 \%$ (3 out of 60) of patients developed EHL weakness and that also recovered with conservative management by the end of 1 year. - The mean ratio of MJS/LJS pre operatively was $0.72 \pm 0.11$ in KSP group while in PFO group it was $0.69 \pm 0.18$. Post operatively the ratio was increased to $1.05 \pm 0.17$ and $0.96 \pm 0.23$ respectively for KSP and PFO group. Ratio of medial and lateral joint space improved in both the groups.

\section{CONCLUSION}

Osteoarthritis of knee joint is a very common health concern in our country and is one of the major causes of locomotor disability in elderly age group. The surgical options are the only viable option till now but are economically unaffordable in developing countries like India and also has lead complications like infection, chronic knee pain, implant failure leading to revision surgeries. The need of hour is to salvage the knee in the early stage of disease especially in relatively younger population to halt the progression of the disease. Since the latest trend is move to regeneration rather than reconstruction, and to keep the knee joint naïve so that TKA can be done, if at all required. Recently, Proximal fibular osteotomy has been emerging as an alternative surgery for management of medial compartment osteoarthritis of knee with promising results. We tried to further increase the efficacy of this procedure by incorporating arthroscopic debridement and leucocyte poor platelet rich plasma (LPPRP) injection to the knee joint with PFO known as Knee Salvation Procedure. In medium to longer term Knee salvation procedure continued to have better outcomes due to supplementation effect of arthroscopic lavage with debridement and LP-PRP. Early weight bearing is possible in both the procedures and no implant insertion is required in any of them. Both the procedures are cost effective, can be readily performed with minimum hospital stay and minimal complications. Patient selection is critical to both the procedures as elderly and patient with high BMI tend to have low satisfaction with these procedures for whom other treatment modalities can be considered.

Lacuna of study: Study needs long term follow up with more number of patients in order to consolidate the results obtained.

Funding- The study did not receive any specific grants from funding agencies in public, commercial or non profit sectors. 
Gourav Mazumdar et.al. Knee salvation procedure (arthroscopic debridement combined with LP-PRP intraarticular injection and proximal fibular osteotomy) vs proximal fibular osteotomy alone in the management of medial compartment knee osteoarthritis: A comparative study of 60 cases.

Declaration of Interest- The authors declare that they have no conflict of interest.

\section{Acknowledgement: None}

\section{Ethical Approval: Approved}

\section{REFERENCES}

1. Di Cesare P, Abramson S and Samuels J (2009) Pathogenesis of osteoarthritis. In: Firestein GS, Kelley WN, eds. Kelley's Textbook of Rheumatology. 8th ed. Philadelphia, Pa.: Saunders Elsevier 2009; 1525-40 2. Woolf AD, Pfleger B. Burden of major musculoskeletal conditions. Bull World Health Organ. 2003;81(9):646-56.

2. Ingemar F Petersson, Torsten Boegård, Tore Saxne, Alan J Silman, Björ n Svensson. Radiographic osteoarthritis of the knee classified by the Ahlbäck and Kellgren \& Lawrence systems for the tibiofemoral joint in people aged 35-54years with chronic knee pain. Annals of the Rheumatic diseases 1997;56:493-496.

3. SM Hussain, DW Neilly, S Baliga, S Patil and RMD Meek: Knee osteoarthritis: a review of management options. Scottish Medical Journal. 2016 Feb;61(1):7-16.

4. Yang ZY, Chen W, Li CX, Wang J, Shao DC, Hou ZY, et al. Medial Compartment Decompression by Fibular Osteotomy to Treat Medial Compartment Knee Osteoarthritis: A Pilot Study. Orthopedics. 2015 Dec;38(12):e1110-4.

5. Dong T, Chen W, Zhang F, Yin B, Tian Y, Zhang Y. Radiographic measures of settlement phenomenon in patients with medial compartment knee osteoarthritis. Clin Rheumatol. 2016; 35(6):1573- 1578.

6. Piuzzi NS, Chughtai M, Khlopas A, Harwin SF, Miniaci A, Mont MA, et al. PlateletRich Plasma for the Treatment of Knee
Osteoarthritis: A Review. J Knee Surg. 2017 Sep;30(7):627- 633.

7. Mirzatolooei F, Alamdari MT, Khalkhali HR . The impact of platelet-rich plasma on the prevention of tunnel widening in anterior cruciate ligament reconstruction using quadrupled hamstring tendon. Bone Joint J 2013; 95-B:65-69 69

8. Mascarenhas R, Saltzman BM, Fortier LA, Cole BJ. Role of platelet-rich plasma in articular cartilage injury and disease. J Knee Surg. 2015 Feb;28(1):3-10.

9. Rönn K, Reischl N, Gautier E, Jacobi M. Current surgical treatment of knee osteoarthritis. Arthritis. 2011;2011:454873

10. Ahlbäck S. Osteoarthrosis of the knee. A radiographic investigation. Acta Radiol Diagn (Stockh). 1968;Suppl 277:7-72.

11. Kellgren JH, Lawrence JS. Radiological Assessment of Osteo-Arthrosis. Ann Rheum Dis. 1957 Dec;16(4):494-502. 52. Wright RW; MARS Group. Osteoarthritis Classification Scales: Interobserver Reliability and Arthroscopic Correlation. J Bone Joint Surg Am. 2014 Jul 16;96(14): 1145-1151.

12. Hefti F, Müller W, Jakob RP, Stäubli HU . Evaluation of knee ligament injuries with the IKDC form. Knee Surg Sports Traumatol Arthrosc.1993.1(3-4): 226-34.

How to cite this article: Mazumdar G, Arora VK, S. K Sharma et.al. Knee salvation procedure (arthroscopic debridement combined with LP-PRP intraarticular injection and proximal fibular osteotomy) vs proximal fibular osteotomy alone in the management of medial compartment knee osteoarthritis: A comparative study of 60 cases. International Journal of Research and Review. 2022; 9(2): 340-350. DOI: https://doi.org/10.52403/ijrr.20220245 